WestVirginiaUniversity
THE RESEARCH REPOSITORY @ WVU

Regional Research Institute Publications and

Working Papers

Regional Research Institute

1996

Exploratory Spatial Data Analysis Linking SpaceStat and ArcView

Luc Anselin

Shuming Bao

Follow this and additional works at: https://researchrepository.wvu.edu/rri_pubs

Part of the Regional Economics Commons 
See discussions, stats, and author profiles for this publication at: https://www.researchgate.net/publication/246146496

\section{Exploratory Spatial Data Analysis Linking SpaceStat and ArcView}

Article · January 1997

DOI: 10.1007/978-3-662-03499-6_3

\section{CITATIONS}

100

2 authors:

Luc Anselin

University of Chicago

232 PUBLICATIONS 28,589 CITATIONS

SEE PROFILE
READS

175

Shuming Bao

University of Michigan

61 PUBLICATIONS 2,039 CITATIONS

SEE PROFILE

Some of the authors of this publication are also working on these related projects:

NIH/Nect $\quad \mathrm{NCI}$ R01 Grant 2-R01CA126858 "Geospatial Factors \& Impacts II" View project

Violence(s)as public health, criminological, and sociological phenomena View project 


\title{
3 Exploratory Spatial Data Analysis Linking SpaceStat and ArcView
}

\author{
Luc Anselin' and Shuming $\mathrm{Bao}^{2}$ \\ I Regional Research Institute and Department of Economics, West Virginia University, \\ Morgantown, WV 26506-6825, USA \\ 2 Data Analysis Products Division, MathSoft, Inc., Seattle, WA 98109, USA
}

\subsection{Introduction}

The extension of the functional capacity of geographic information systems with tools for spatial analysis has been an increasingly active area of research in recent years. Following Goodchild's (1987) call to action [see also Goodchild (1992)], a growing number of conferences and workshops has been devoted to the topic in the academic GIS community, resulting in many articles and several edited volumes [among others, Fischer and Nijkamp (1993), Fotheringham and Rogerson (1994), Painho (1994), Fischer, Scholten and Unwin (1996)]. Among the types of analyses suitable for inclusion within the functionality of a GIS, spatial data analysis in particular has received considerable attention. Several conceptual frameworks have been suggested, proposing different degrees of linkages between GIS and spatial analysis, and outlining the types of spatial statistical techniques that would be most suitable for inclusion [examples are Openshaw (1991), Anselin and Getis (1992), Goodchild et al. (1992), Bailey (1994), Haining (1994), Openshaw and Fischer (1995)]. In broad terms, the proposed linkages can be described as either tight vs. loose [Goodchild et al. (1992)] or as encompassing vs. modular [Anselin and Getis (1992)] depending on the degree to which a module with "traditional" GIS functionality and a data analysis module are integrated into a single software environment.

While the early discussions stressed conceptual frameworks and taxonomies of techniques, lately several operational implementations of these ideas have been carried out as well. Early such efforts followed what Anselin et al. (1993) call a onedirectional form of integration, in which data from a GIS are efficiently transferred to a statistical system for analysis, or results from a statistical package are moved to the GIS for mapping and visualization. Such attempts typically follow the loosecoupling or modular paradigm. Early illustrative examples of this approach, among many others, are the joint use of the Grass GIS with the S statistical package to carry out exploratory data analysis in Farley et al. (1990), and Williams et al. (1990), and the use of Poisson regression results from Glim as a basis for areal interpolation in the Arc/Info GIS in Flowerdew and Green (1991). More recently, a close coupling approach was outlined in Symanzik et al. (1994), in which information from the Arc/Info GIS is efficiently passed to the XGobi software environment for exploring multivariate data by means of dynamic graphics, brushing, linking and the grand tour [for a recent review of the XGobi environment, see Buja et al. (1996)]. However, 
while sophisticated in its implementation, the link remained one-directional, in the sense that all analysis and further data visualization is carried out in XGobi and the results are not passed back to the GIS.

A dynamic integration necessitates a software environment in which spatial data and the results of spatial data analyses efficiently move back and forth between the GIS and a statistical module to allow a truly interactive data analysis [Bailey and Gatrell (1995), Anselin (1997a)]. A prototype for such a dynamic integration was suggested in Anselin et al. (1993), consisting of Arc/Info as the GIS module and SpaceStat [Anselin $(1992,1995 a)$ ] as the spatial data analysis module. This implementation was based on a loose coupling or modular approach, in which data (or results) are passed between the two modules using auxiliary files with standardized file names, to minimize the amount of user intervention in the process. A similar framework is outlined in Zhang et al. (1994), where four software modules are linked in a loose fashion across both workstation and PC platforms, to allow GIS processing (in Arc/Info), cluster analysis (Mclust-Plus), traditional exploratory data analysis (XGobi) as well as spatial data analysis (SpaceStat).

Most recent implementations of dynamic integration have not been based on loose coupling, however, but have focused instead on extending the functionality of existing commercial GIS with spatial data analysis routines, by taking advantage of the system's macro or scripting language facilities (e.g., AML for Arc/lnfo) as well as by calling pre-compiled functions. This approach is fully integrated within the GIS user interface and typically hides the linked nature of the analysis routines from the user. Recent examples are the extension of Arc/Info with functions for the implementation of non-spatial EDA tools, such as scatterplots [e.g., Batty and Xie (1994)], as well as routines for the computation of global and local indicators of spatial autocorrelation [Ding and Fotheringham (1992), Bao et al. (1995), Can (1996)]. A well-recognized drawback of this integrated approach is a performance penalty, since the macro or scripting languages are not optimized to handle the computations necessary for spatial statistical analysis, resulting in a loss of speed and/or limitations on the size of the problems that can be handled. An alternative to this that retains the close-coupling paradigm is the linkage between two software packages that allow remote procedure calls (in unix) or dynamic data exchange (in a Windows environment). This approach is taken in the only commercial implementation that exists to date of an integrated data analysis and GIS environment, the $\mathrm{S}+\mathrm{Gislink}$ between the S-Plus statistical software and the Arc/Info GIS. In the S+Gislink add-on to the S-Plus software for unix workstations, a bidirectional link is established with the Arc/Info GIS that allows data to be imported and exported between the two packages in the native data formats (i.e., the internal data frame format in S-Plus and the Info data table in Arc/Info). In addition, the linkage allows users to call S-Plus functions from within Arc/Info, although the reverse is not possible [MathSoft (1996a)]. A similar perspective is taken in the recent efforts at the Statistics Laboratory of Iowa State University to implement a linked environment between ArcView and XGobi on unix workstations, focused on the use of XGobi for exploratory data analysis, such as the visualization and brushing of scatterplots and cumulative distribution functions [e.g., Majure et al. (1996), Symanzik et al. (1996), Cook et al. (1996), Majure and Cressie (1997)]. 
In this chapter, we extend and further operationalize the framework of Anselin et al. (1993), using the ArcView GIS [ESRI (1995a)] in a Windows environment as the visualization engine, and Spacestat as the spatial data analysis engine. Our approach differs from the efforts described above in three respects: (a) it has an explicit focus on the so-called lattice perspective towards spatial data analysis, in contrast to the geostatistical viewpoint that currently dominates in the literature; (b) it uses the GIS (ArcView) for the visualization of the statistical results, not the statistical software (compared to the use of respectively S-Plus and XGobi for visualization in the $\mathrm{S}+$ Gislink and Iowa State implementations); and (c) it is primarily targeted at a PC platform, although in the right network environment, it could be implemented in unix as well (ArcView exists for both unix and Windows platforms)

In terms of methodology, our emphasis is on tools for exploratory spatial data analysis (ESDA) in which the spatial dependence of the data is taken into account explicitly. This contrasts with most other implementations of linked EDA and GIS, where traditional EDA tools such as box plots, histograms and scatterplots are the focus of attention and the complicating effects of spatial autocorrelation are typically ignored [e.g., MathSoft (1996b, Chapter 3)].

In the remainder of the chapter, we first define our vision of ESDA and outline the types of techniques that are central to it. This is followed by a more detailed conceptual overview of the linkage between spatial data analysis and GIS and its implementation for ArcView and SpaceStat. We illustrate this framework with a few examples in which the spatial pattern of housing values in West Virginia counties is examined. We close with some remarks on future directions.

\subsection{Exploratory Spatial Data Analysis}

Exploratory Data Analysis, or EDA, has become increasingly popular as a methodology to generate insight into patterns and associations in data (especially large data sets), without strong prior assumptions and taking into account the potentially misleading influence generated by "extreme" or "atypical" observations. Since the pioneering work of Tukey (1977), EDA has gained considerable influence as a paradigm in applied statistics and it now forms the basis for many of the visualization and graphical features of modern statistical software [Good (1983), Cleveland (1993), Venables and Ripley (1994)]. However, none of the traditional tools of EDA are especially geared to dealing with spatial data, in the sense that the effects of location, spatial dependence and spatial heterogeneity are ignored. Moreover, many EDA techniques for the initial exploration of bivariate and multivariate relationships, such as smoothed scatterplots, may yield indications that are invalid in the presence of spatial autocorrelation [Anselin (1990), Anselin and Getis (1992)]. In contrast, Exploratory Spatial Data Analysis (ESDA), focuses explicitly on these spatial effects and consists of techniques to describe spatial distributions, identify atypical locations (spatial outliers), discover patterns of spatial association (spatial clustering) and suggest different spatial regimes or other forms 
of spatial instability (spatial non-stationarity) [Anselin (1994)]. We follow Anselin (1994) in drawing the distinction between true ESDA and so-called spatialized EDA, in which standard EDA features are displayed at particular locations on a map, or shown as a function of a given distance metric. Familiar examples of the latter are the mapping of Chernoff faces in two-dimensional space [e.g., Haining (1990, p. 226), Fotheringham and Charlton (1994)], or the display of box plots by distance bands [Haining (1990, p. 212, 224)].

Central to ESDA is the concept of spatial association or spatial autocorrelation. In this respect, it is important to draw a distinction between methods appropriate for the geostatistical or distance-based perspective on the one hand and those geared to the lattice or neighborhood view of spatial data on the other hand [see Cressie (1993), Anselin (1994)]. Our focus will be on the latter, in which data are observed for a given discrete set of fixed locations. In contrast to the geostatistical perspective, these locations are not considered to form a sample of an underlying continuous distribution, but the data are conceptualized as a single realization of a spatial stochastic process, similar to the approach taken in time series analysis. In this view, spatial interaction is conceptualized as a step function, where a location interacts with a given set of neighbors. The overall interaction (or covariance) in the observed data is then obtained by imposing (assuming) a particular form for the spatial stochastic process. This approach requires the formal expression of a neighborhood structure for each observation (i.e., the topology or spatial arrangement of the data) in the form of a spatial weights matrix $\mathbf{W}$. In this matrix, nonzero elements indicate the presence of a neighbor relationship, which may be expressed as a simple binary variable (e.g., $\mathrm{w}_{\mathrm{ij}}=1$ ) or may take on a more general form, as a prior for the strength of interaction between observation $i$ and its neighbor $j$. By convention, the diagonal elements of the weights matrix $\left(\mathrm{w}_{\mathrm{ii}}\right)$ are set to zero [for a more extensive discussion, see, e.g., Cliff and Ord (1981), Upton and Fingleton (1985), Anselin (1988)]. For realistically sized problems, the construction of such a weights matrix cannot be carried out by visual inspection of a map and must rely on the data structures present in a GIS [see, e.g., Anselin (1995a)].

In the traditional approach to spatial autocorrelation, the overall pattern of dependence in the data is summarized into a single indicator, such as the familiar Moran's I, Geary's c or Gamma indicators of spatial association [for details, see, e.g., Cliff and Ord (1981), Upton and Fingleton (1985), Haining (1990)]. We refer to this as global spatial autocorrelation, in contrast to local indicators of spatial association (LISA) which we consider below. The various global measures of spatial association can be used to assess the range of spatial interaction in the data and can be easily visualized by means of a spatial correlogram (a series of spatial autocorrelation measures for different orders of contiguity) [see, e.g., Oden (1984), and for a recent application, Lam et al. (1996)]. A major drawback of global indicators of spatial association is that they are based on an assumption of spatial stationarity, which among other requirements necessitates a constant mean (no spatial drift) and constant variance (no outliers) across space. While this may have been useful in the analysis of small data sets, such as in the classic example of 26 Irish counties in Cliff and Ord (1981), it is not very meaningful or may even be highly misleading in analyses of spatial association for hundreds or thousands of spatial units that characterize current 
GIS applications. In addition, most of these global measures of spatial association were developed in an era of scarce computing power, small data sets and minimal computer graphics, and their implementation takes only limited advantage (if at all) of the data storage, retrieval and visualization capabilities embodied in a modern GIS. Hence, rather than stressing these global statistics as useful additions to the analytical capabilities of a GIS [as in Griffith (1993)], we see the main contribution of ESDA techniques in measuring and displaying local patterns of spatial association, indicating local non-stationarity and discovering islands of spatial heterogeneity [Anselin (1994)].

In the subsections below, we elaborate on three classes of ESDA techniques that are implemented in the linked SpaceStat-ArcView framework outlined in section 3: the description and visualization of spatial distributions; the visualization of global spatial association and detection of spatial non-stationarity; and local indicators of spatial association. The illustration of these techniques is deferred to section 4 .

\subsubsection{Describing Spatial Distributions}

The description of spatial distributions has become increasingly integrated within the interactive and dynamic visualization techniques of EDA, such as scatterplot brushing [Becker and Cleveland (1987)] and plot windows [Stuetzle (1987)], in which multiple views of the data (such as tables, charts and plots) are presented simultaneously. The views are shown in different windows on a computer screen, and are dynamically linked in the sense that when a location in any one of the windows (e.g., a bar on a bar chart, or a set of points in a plot) is selected by means of a pointing device (socalled brushing), the corresponding locations in the other windows are highlighted. This allows a highly interactive approach to data analysis, which is particularly effective in detecting unexpected patterns in high-dimensional data [Buja et al. (1991, 1996)].

While geographic locations have always played an important role in dynamic graphics [e.g., the various examples in Cleveland and McGill (1988)], it is only recently that the map was introduced explicitly as an additional view on the data, e.g., in Monmonier (1989), Haslett et al. (1990) and MacDougall (1991). Particularly in the Spider-Regard software tools of Haslett, Unwin and associates, the distribution of data in a spatial subset of observations can be effectively visualized by means of a linked map, histogram and box plot [for a recent overview, see Unwin (1994)]. In other words, for any subset of locations highlighted on the map, the corresponding distribution of the data is highlighted in a histogram and/or box plot and can thereby be contrasted to the overall distribution. Also, for any subset of data highlighted in a non-spatial view, such as the histogram, the corresponding locations are highlighted on the map.

A slightly different approach towards visualizing the distribution of spatial data is by means of a spatial cumulative distribution function (SCDF). In the implementation of this idea in Majure et al. (1996) and Cook et al. (1996), a continuous density function is estimated for all observations in a given region. By linking a map in ArcView and the SCDF plot in XGobi, it becomes possible to 
highlight subregions of the data and to find the corresponding portion of the SCDF plot, and vice versa.

When spatial data pertain to aggregate areal units, it is straightforward to visualize the distribution of a variable by means of a quantile map, i.e., a choropleth map in which each color corresponds to observations within a given quantile of the spatial distribution. For a quartile map (four quantiles), this matches the grouping of observations in a box plot, except that the latter also indicates outliers in the distribution as locations outside the "fences" in the plot [see Cleveland (1993, pp. 25-27)]. It is straightforward to construct an equivalent device in the form of a $b o x$ map, in which the quartile map is augmented with highlighted outliers (e.g., in different colors for the lower and upper outliers). The comparison of box maps for different variables provides an initial look at potential multivariate associations, in the sense that maps with matching quartiles (and outliers) are likely to correspond to correlated variables [e.g., Talen (1997)].

In terms of the integration of GIS with spatial data analysis to describe spatial distributions, it becomes clear that the capacity to dynamically link different views of the data is crucial. This is further explored in section 3 and illustrated in section 4.

\subsubsection{Visualizing Patterns of Spatial Association}

The typical approach towards visualizing spatial dependence taken in the literature is based on the geostatistical perspective and uses the variogram as a parameter of spatial association. The basic tools are outlined in Cressie (1993) and include a variogram cloud (a scatterplot of squared differences between pairs of observations, sorted by distance band), a variogram box plot (a box plot of the variogram cloud for each distance band) and a spatial lag scatterplot (a scatterplot for a given distance band where the horizontal axis corresponds to the value at each location and the vertical axis shows the corresponding value at the spatially lagged locations). The variogram cloud is integrated with the dynamically linked windows in the SpiderRegard software system to investigate patterns of spatial association in subsets of the data (e.g., by brushing points in the variogram cloud, the corresponding locations on the map are highlighted), with a special focus on detecting local "pockets" of spatial non-stationarity [see, e.g., Haslett et al. (1991), Haslett (1992), Bradley and Haslett (1992), Haslett and Power (1995)]. A recent extension of the use of the spatial lag scatterplot to a multivariate setting is given in Majure and Cressie (1997).

When data consist of aggregate areal units, i.e., when the lattice or neighborhood view is taken, the variogram is less meaningful as a device to model spatial dependence, since it relies on the assumption of an underlying continuous spatial process. Instead, a crucial role in the visualization of spatial association is played by the concept of a spatial lag. In Anselin (1988), this is defined as a weighted average of the values observed for the neighbors of a given location, where the weights are taken from a spatial weights matrix. More formally, when $w_{i j}$ are row-standardized spatial weights (i.e., such that $\Sigma_{\mathrm{j}} \mathrm{w}_{\mathrm{ij}}=1$ ), the spatial lag for $\mathrm{y}_{\mathrm{i}}$ would be $\Sigma_{\mathrm{j}} \mathrm{w}_{\mathrm{i} \cdot \mathrm{j}} \mathrm{y}_{\mathrm{j}}$. The structure of the weights matrix ensures that only those values of $y_{j}$ are taken into 
account for which the locations $j$ are neighbors to $i$ (since $\mathrm{w}_{\mathrm{ij}}=0$ for locations that are not neighbors). In matrix notation, the vector of observations on a spatially lagged variable consists of the product of the spatial weights matrix with the vector of observations, W.y.

The matching of a value observed at each location with its spatial lag provides useful insight into the local as well as the global pattern of spatial association in the data. Specifically, when a high degree of positive local spatial autocorrelation is present, the observed value at a location and its spatial lag will tend to be similar. In the extreme case, the value at a location would be predicted exactly by the observed values in the neighboring locations (implying a spatial autoregressive coefficient of 1). In a global sense, spatial clusters would be indicated by subsets of locations with great similarity between the values observed and their spatial lags. In the opposite situation, when a high degree of negative spatial autocorrelation is evident, low values at a location would tend to be surrounded by higher values for the neighbors (i.e., a higher weighted average for the neighbors compared to the value at the location), or high values would tend to be surrounded by lower values for the neighbors. If the magnitude of the difference is sufficient, both instances could be classified as spatial "outliers." The presence of many spatial outliers intermixed with an overall pattern of positive spatial association may provide evidence of local nonstationarity.

The association between a variable and its spatial lag is easily visualized by means of so-called spatial lag pies and spatial lag bar charts [Anselin et al. (1993), Anselin (1994)]. The former are only appropriate when the observed values are all strictly positive, since they rely on the division of a circle into two pies, each of which is proportional to the relative share of the variable and its spatial lag in their sum. More precisely, the share corresponding to each pie is $y_{i} /\left[y_{i}+(W y)_{i}\right]$ and $(\mathrm{Wy})_{\mathrm{i}} /\left[\mathrm{y}_{\mathrm{i}}+(\mathrm{Wy})_{\mathrm{i}}\right]$. Hence, for $\mathrm{y}_{\mathrm{i}}=(\mathrm{Wy})_{\mathrm{i}}$, each pie would equal half the circle. Negative spatial autocorrelation for high values would be indicated by the dominance of the pie share corresponding to $y_{i}$ and vice versa. The circles themselves may be graduated, to indicate the overall magnitude of the value observed at each location [for an illustration, see Anselin et al. (1993)]. In a spatial bar chart, the graph simply consists of two bars at each location, one of which corresponds to $y_{i}$, the other to $(\mathrm{Wy})_{\mathrm{i}}$. Other visualization schemes are possible as well, for example, based on the difference, absolute difference, squared difference or ratio between $\mathrm{y}_{\mathrm{i}}$ and $(\mathrm{Wy})_{\mathrm{i}}$ at each location. The spatial lag bar chart is illustrated in section 4 .

A more quantified approach towards visualizing local and global spatial association is based on the concept of a Moran scatterplot [Anselin (1995b, 1996)]. It follows from the interpretation of the familiar Moran's I statistic for spatial autocorrelation as a regression coefficient in a bivariate spatial lag scatterplot. More precisely, for a row-standardized weights matrix, the normalizing constants in Moran's $I$ cancel out and the statistic reduces to $I=z^{\prime} \mathrm{Wz} / \mathrm{z}$ ' $\mathrm{z}$, with $\mathrm{z}$ as the vector of observations in deviations from the mean. This is the slope coefficient in a regression of Wz on $\mathrm{z}$. The interpretation of Moran's I as a regression coefficient has three interesting implications. First, the statistic can easily be visualized as the slope of a straight line in a scatterplot, which is especially insightful when the pattern of spatial association is evaluated for different variables, or for the same variable over 
time [for an example, see O'Loughlin and Anselin (1996)]. Secondly, since the statistic is obtained from least squares estimation, the usual battery of diagnostics may be applied to identify outliers and observations with high leverage or influence on the slope. This often provides an intuitive check for border effects and other potential consequences of a poorly specified spatial weights matrix [for technical details, see Anselin (1996)]. Thirdly, the extent to which more general nonlinear scatterplot smoothers, such as a loess regression, provide a different fit than the linear regression may suggest spatial regimes and other forms of non-stationarity.

A second use of the Moran scatterplot is as a device to decompose the global spatial autocorrelation statistic into four types of association. These four types correspond to the four quadrants in the scatterplot when the variable $\mathrm{z}$ is normalized, such that its mean is zero and its standard deviation equals one. The upper right and lower left quadrants correspond to instances of positive spatial association [in the traditional sense of Cliff and Ord (1981)], i.e., the presence of similar values in neighboring locations. For the upper right quadrant, this association is between values of $z_{i}$ above the mean whose spatial lag is also above the mean, and in the lower left quadrant, these are values of $z_{i}$ below the mean whose spatial lag is also below the mean. The other two quadrants correspond to negative spatial association, values of $z_{i}$ below the mean whose spatial lag is above the mean (upper left quadrant), and values of $z_{i}$ above the mean whose spatial lag is below the mean (lower right quadrant). Both of these could indicate potential spatial outliers, provided that the values involved are "extreme" enough. The latter is easy to assess, since the scatterplot is constructed for standardized variates. Consequently, two units on the scatterplot correspond with two standard deviations from the mean, which can be used to identify outliers as those points outside a circle with radius equal to two that is centered on the origin. The decomposition of the global spatial association into the four quadrants of the Moran scatterplot can also be mapped in a straightforward fashion, with each quadrant corresponding to a different color or shading on the map (possibly only for those points identified as outliers). The resulting Moran scatterplot map is illustrated in section 3.4.

\subsubsection{Local Indicators of Spatial Association}

More recently, in part to address the need to develop techniques to analyze the large spatial data bases that are becoming increasingly available, attention has focused on local indicators of spatial association, or LISA [Getis and Ord (1992), Anselin (1995b), Ord and Getis (1995), Bao and Henry (1996), Unwin (1996)]. Following the definition of Anselin (1995b), a LISA is an indicator that achieves two objectives: (a) it allows for the detection of significant patterns of local spatial association (i.e., association around an individual location, such as hot spots and spatial outliers); and (b) it can be used as a diagnostic for stability of a global diagnostic (i.e., to assess the extent to which the global pattern of association is reflected uniformly throughout the data set). Not all local statistics suggested in the literature fit the two requirements. For example, the $G_{i}$ and $G_{i}{ }^{*}$ statistics of Getis and Ord (1992) primarily satisfy the first objective, while the Moran scatterplot discussed in the previous section is geared 
to the detection of local "pockets" of non-stationarity in the computation of Moran's I.

The first indicators of local spatial association that gained wide acceptance were the distance-based $G_{i}$ and $G_{i}{ }^{*}$ statistics of Getis and Ord (1992). These indicators can be computed for each location in the data set as the ratio of the sum of values in neighboring locations (defined to be within a given distance band or order of contiguity) to the sum over all the values. The two statistics differ with respect to the inclusion of the value observed at $i$ in the calculation (not included for $\mathrm{G}_{i}$ ). Formally, a $G_{i}$ statistic is thus $\Sigma_{j} w_{i j}(d) y_{j} / \Sigma_{j} y_{j}$, with $w_{i j}(d)$ as a distance-based binary spatial weights matrix. Locations with a statistically significant $\mathrm{G}_{i}$ or $\mathrm{G}_{\mathrm{i}}{ }^{*}$ statistic can easily be mapped and used in an exploratory analysis to detect hot spots or spatial clusters [e.g., Ding and Fotheringham (1992), Anselin et al. (1993), Bao et al. (1995), O'Loughlin and Anselin (1996), Unwin (1996)]. The interpretation of the statistics is slightly different from standard practice in spatial autocorrelation analysis, in the sense that a negative statistic points to association between similar small values (and not to association between dissimilar values). Consequently, the $G_{i}$ and $G_{i}{ }^{*}$ statistics measure association in the upper right and lower left quadrants of the Moran scatterplot, but not in the others. A global Moran's I statistic can be expressed as a weighted average of $\mathrm{G}_{i}{ }^{*}$ statistics [Ord and Getis (1995)].

The local Moran statistic outlined in Anselin (1995b) satisfies both criteria for a LISA and can thus effectively be used to identify both local spatial clusters as well as to assess the stability of the global Moran's $I$. The former use is more appropriate when no global association is found in the data, while the latter is more appropriate in the opposite case. When local Moran statistics are computed in the presence of global spatial autocorrelation, their significance must be interpreted with caution [see Anselin (1995b), Ord and Getis (1995), for technical details] although they remain useful as an exploratory technique. Formally, the local Moran statistic for observation $i$ is $z_{\mathrm{i}}\left[\Sigma_{\mathrm{j}} \mathrm{w}_{\mathrm{ij}} \cdot \mathrm{z}_{\mathrm{j}}\right]$, where $\mathrm{w}_{\mathrm{ij}}$ are the elements of a spatial weights matrix and the $\mathrm{z}_{\mathrm{i}}$ are standardized variates. Significance of the local Moran can be derived analytically under the null hypothesis of no spatial association, or by means of a conditional permutation approach [for technical details, see Anselin (1995b), Bao and Henry (1996)]. The local Moran is particularly useful as an ESDA tool when used in conjunction with a Moran scatterplot. Specifically, the quadrant in a Moran scatterplot indicates what type of spatial relationship is found for locations with significant local Moran statistics. The mapping of significant LISA statistics in a GIS, together with a Moran scattermap and/or overlays of maps for other variables of interest provides the basis for a substantive interpretation of spatial clusters or spatial outliers [for examples, see Barkley et al. (1995), Talen and Anselin (1997)]. This is further illustrated in section 4 .

\subsection{Linking Exploratory Spatial Data Analysis and GIS}

The linkage between exploratory spatial data analysis and GIS considered here builds upon the general framework outlined in Anselin and Getis (1992). Following the 


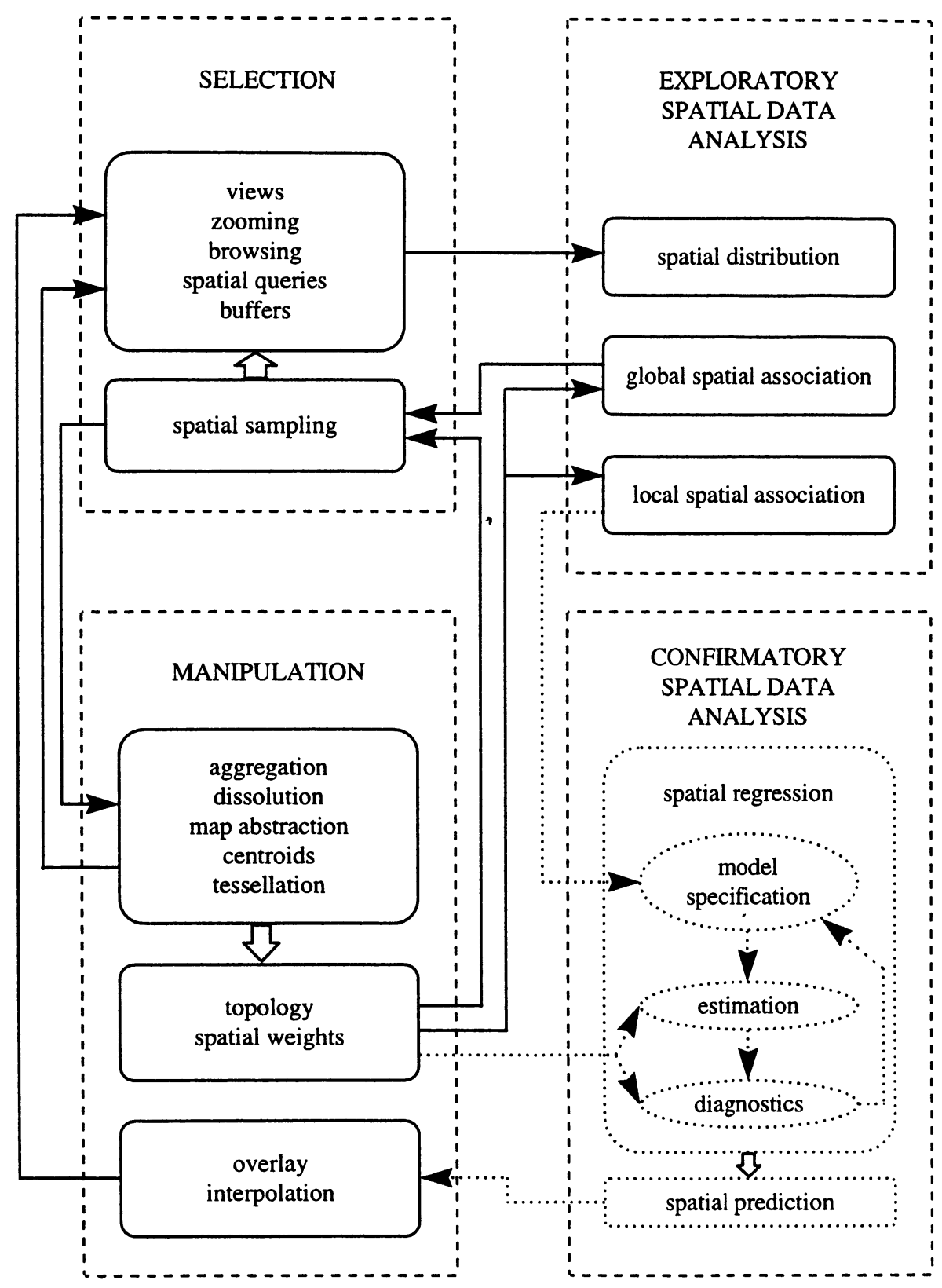

Fig. 3.1. Integration of GIS Module with Spatial Data Analysis Module 
usual classification of GIS functionality into four broad groups - input, storage, analysis and output - they further subdivided the analysis function into selection, manipulation, exploration and confirmation. In Anselin et al. (1993), the first two of these functions were considered to form a "GIS module" while the latter two formed a "Data Analysis module" to emphasize the practical division of labor between typical commercial GIS software and the specialized (add-on) software needed to carry out spatial data analysis. This distinction has become increasingly tenuous, since many statistical software packages now have some form of mapping (or even GIS) functionality, and a growing number of statistical functions are included in GIS software. More important than classifying these functions as belonging to one or the other module is to stress their interaction and the types of information that must be interchanged between them.

A schematic overview of the linkages between the four functions is given in Figure 3.1, with an emphasis on the links between the selection and manipulation functions and exploratory spatial data analysis [for a more extensive discussion of the various linkages, see also Anselin (1997b)]. While many taxonomies are possible, the main point of the classification in Figure 3.1 is that selection and manipulation (shown on the left) are present in virtually all systems and have become known as "spatial analysis" in the commercial world [e.g., ESRI (1995b, Lesson 8)]. In contrast, the spatial data analysis functions (shown on the right) are much less prevalent in commercial systems.

The selection functions include operators necessary to obtain the values (attributes) of a set of variables for particular locations in a spatial data base. This ranges from simple zooming and browsing functions and traditional relational data base queries to spatial queries, buffering and spatial selection. This is the starting point for any ESDA and is particularly relevant for the computation and display of the distribution of values over a given spatial subset of locations (spatial density functions, box map). Related to this is a spatial sampling function, in which a subset of locations is selected to represent a spatial "population" in further statistical analysis. Due to the prevalence of spatial autocorrelation, standard random sampling techniques may not be appropriate for spatial data sets. In order to carry out proper spatial sampling, often an initial data analysis is needed to assess the range and significance of spatial autocorrelation. This would be the result of an exploratory spatial data analysis, as illustrated by the link between global spatial association and spatial sampling in Figure 3.1.

The manipulation function includes all operations to "create spatial data." The virtual limitless ability of GIS to produce maps of data at any scale and for any level of areal aggregation is often seen as its most powerful "analysis" feature. However, though typically hidden from the user, such operations are themselves based on specific functions, algorithms and models and often involve a prior statistical sampling and/or analysis of the data. The data manipulation operations can be broadly classified into three groups. The first contains those pertaining only to attribute values, i.e., traditional data summaries and transformations (aggregation, averaging, etc.). A second group consists of those operations that pertain only to spatial information, i.e., a manipulation of the coordinates of the points, lines and polygons in a spatial data base to perform spatial transformations, map abstraction, 
spatial aggregation and dissolution, the computation of topology (determination of neighbors), centroids, area, perimeter, etc. The most important aspect of this second group for data analysis is the construction of topology or spatial arrangement for a set of areal units. This information is crucial for the computation of any statistic for spatial autocorrelation, for which a spatial weights matrix or spatial lag operator is essential. As pointed out earlier, the spatial lag is a central element in the visualization and exploration of both global and local spatial association (in the ESDA module). Finally, a third group of functions combines both spatial and nonspatial information and is commonly referred to as "data integration." This capability allows for the construction of "data" for a particular unit of analysis by combining information on different variables and at different levels of spatial aggregation by means of polygon overlay and spatial interpolation operations. The flexibility to move between different levels of spatial aggregation, to relate multiple variables in a spatial data base and to interactively select subsets of observations provides a powerful platform to carry out ESDA. The particular implementation of these linkages between the ArcView GIS and the SpaceStat spatial data analysis software are considered in more detail next.

\subsubsection{Linking SpaceStat and ArcView}

The principle behind the linkage of ArcView (Version 2.1) and SpaceStat (Version 1.80) to facilitate exploratory spatial data analysis is the transfer of spatial information from ArcView to SpaceStat for analysis, and the transfer of locationspecific results from SpaceStat to ArcView for visualization. The spatial information consists of both location and topology (spatial arrangement) of the selected data points (or areal units). The particular ESDA results considered here include spatial lags (for spatial lag pies and spatial lag bar charts), quartiles and outliers (for a box map), the quadrants in a Moran scatterplot (for a Moran scatter map), and the significant LISA and $\mathrm{G}_{i}$ statistics. However, any statistic that has a value assigned to each location in the data set (e.g., observations, regression residuals and predicted values) can be efficiently passed back from SpaceStat to ArcView for visualization using the same principles as outlined in what follows.

The division of labor implemented here is different from the approach taken in Majure et al. (1996), Symanzik et al. (1996) and Cook et al. (1996), who also link ArcView with a statistical package (XGobi). There, ArcView is primarily used to select spatial subsets of locations and the visualization is an output of the XGobi software. The primary aspect of their linkage is to highlight "brushed" data points in both the map (in ArcView) and the various types of scatterplots in XGobi. However, since their ESDA is based on a geostatistical perspective, the topology of the data embedded in the GIS does not need to be exploited. In contrast, this is a central element in the ESDA carried out in SpaceStat, which is based on the use of spatial weights and spatial lags.

The linkage between ArcView and SpaceStat is not dynamic, but based on a loose coupling approach. In part, this is necessitated by the fact that SpaceStat (still) runs under Dos, whereas ArcView is a 32 bit Windows product. While the new MS 


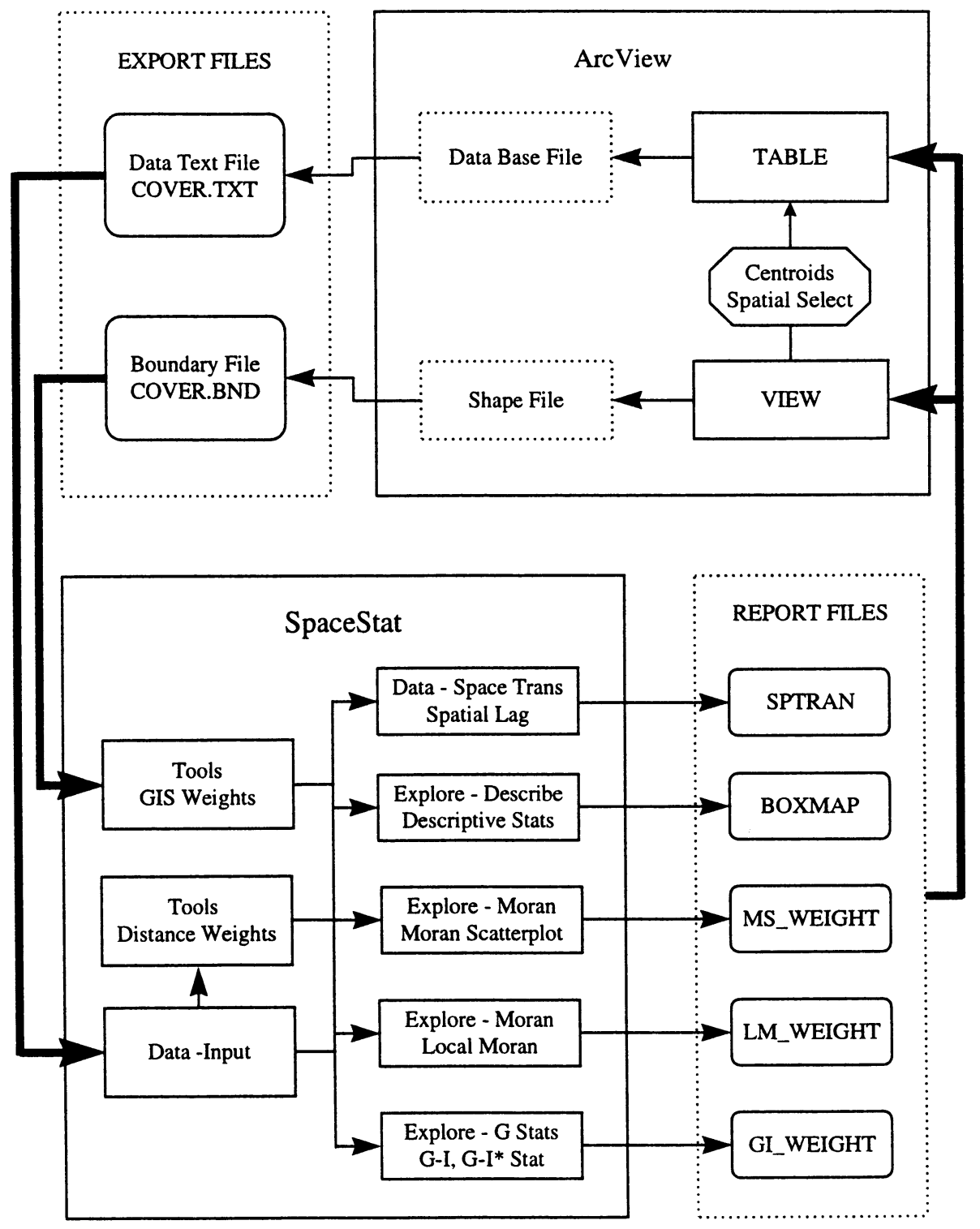

Fig. 3.2. Linkage of SpaceStat and ArcView 
Windows platforms (Windows NT and Windows 95) allow SpaceStat to multi-task with ArcView and run "simultaneously" in a separate window, there is no mechanism to call internal SpaceStat functions from within ArcView, the way this is possible through DDE (dynamic data exchange) and/or OLE (object linking and embedding) between true windows programs (in unix, the same effect is achieved by means of remote procedure calls). However, the amount of additional work is minimized by exploiting the structure of the files used in the data exchange between ArcView and SpaceStat, and in most instances it is reduced to a single click on a menu item or windows toolbar button.

A schematic overview of the linkage between ArcView and SpaceStat is given in Figure 3.2. The typical point of departure is ArcView (in the upper right hand corner) in which both a map view (the "View") and a tabular view (the "Table") of spatial data is standard. These standard features can be supplemented by a few specialized functions that operate on the View and extract or construct spatial "variables" (fields) for addition to the attribute table associated with the View. Specifically, "spatial selection" creates an indicator variable that takes on a value of one for the selected spatial units. The selection itself can be carried out interactively with the map, using the standard ArcView selection tools (or some additional tools that can easily be constructed, see section 3.2). The resulting indicator variable can be used to create subsets of data sets and spatial weights in SpaceStat, or form the basis for a spatial analysis of variance or for spatial regimes in the study of spatial heterogeneity. Another pair of important spatial attributes of a view are the $\mathrm{x}$ and $\mathrm{y}$ coordinates that correspond to the centroids of the (selected) areal units. These coordinates are the point of departure for the construction of distance-based spatial weights in SpaceStat.

Every Table in ArcView is implemented as a data base file that can be converted to an ascii text format in a straightforward manner. This is the first type of export file that will be used to link the data in ArcView to a SpaceStat data set (upper left hand corner in Figure 3.2). The second type contains the information needed to construct the topology or spatial arrangement of the areal units in the View. While ArcView is not a "topological" GIS, in the sense that the left-right polygon topology of the arcs in the map is not recorded explicitly, the data format of the "shape files" is public and can be exploited to build the topology [ESRI (1995c)]. This is implemented by means of an existing SpaceStat utility that reads the binary shape file and converts it to a standard boundary file in ascii format [see Anselin (1995a) for technical details].

In SpaceStat, the two export files from ArcView are converted into data files and spatial weights by means of the functions Data - Input and Tools - GIS Weights (lower left corner of Figure 3.2). Once the $\mathrm{x}$ and $\mathrm{y}$ coordinates of the centroids are contained in a SpaceStat data set, they can be used to build distance-based spatial weights with the corresponding function in the SpaceStat Tools module. The spatial weights can further be row-standardized, higher order contiguity can be constructed and several other manipulations can be carried out in the SpaceStat Tools module. The data and spatial weights are used in a wide range of exploratory and confirmatory spatial data analyses in SpaceStat [for a detailed description, see Anselin (1992, 1995a)]. Specifically, one data transformation and four types of exploratory analyses can be efficiently linked with ArcView by means of a SpaceStat Report File: spatial lag transformation for visualization of spatial autocorrelation (Data - Space Trans, 
Spatial Lag), quartiles with outliers (Explore - Describe, Descriptive Stats), Moran scatterplot (Explore - Moran, Moran Scatterplot), LISA-local Moran (Explore Moran, Local Moran), and $\mathrm{G}_{i}$ and $\mathrm{G}_{i}{ }^{*}$ statistics (Explore - $\mathrm{G}$ Stats, $\mathrm{G}_{\mathrm{i}}$ or $\mathrm{G}_{\mathrm{i}}{ }^{*}$ ). The Report File is a comma delimited ascii file with a distinctive file name and containing the values of an indicator variable as its first column (lower right hand corner of Figure 3.2). This indicator variable is exploited to join the Report File with the attribute table of an active View in ArcView, with minimal user input. Once the Report Files are joined, the relevant variables in them can be visualized in the View in a direct manner.

While the data input and report file output are standard features of SpaceStat, the data export and table join aspects of the linkage in ArcView require some customization of the software. We next turn to the specifics of the operational implementation of the interface in ArcView.

\subsubsection{Operational Implementation of the Interface in ArcView}

As illustrated in Figure 3.2, the essence of the linkage between ArcView and SpaceStat consists of the creation of export files from attribute tables, and the importing and joining of SpaceStat Report files with existing attribute tables. Both of these tasks are carried out within ArcView. They are implemented by means of a collection of customized scripts in the Avenue object oriented macro language [ESRI (1994)], and by the extension of the standard ArcView user interface with two additional menus and a few extra buttons and tools [the collection of scripts is available as the SpaceStat.apr project file from the SpaceStat web site at http://spacestat.rri.wvu.edu; for a technical description, see Anselin and Bao (1996)].

\begin{tabular}{l}
\hline Data Explore Window Help \\
Add Centroid Coordinates \\
\hline Add Selected Features Dummy \\
\hline Weights from Shape File \\
\hline Export Table as Text File \\
Import Table from Text File \\
Join SpaceStat Report File \\
\hline
\end{tabular}

Fig. 3.3. Contents of Data Menu

The menus, buttons and tools are added to the standard View interface in ArcView. The Data menu (Figure 3.3) consists of six commands divided into three categories: (a) the computation of "spatial" variables, i.e., centroid coordinates (Add Centroid Coordinates) and indicator variables for spatial selection (Add Selected Features Dummy); (b) the conversion of shape files to ascii boundary files Weights from Shape File); and (c) the linking of data tables, either the conversion of attribute tables to text format for later input into SpaceStat (Export Table as Text File), importing any 


\begin{tabular}{|c|c|}
\hline Data & Explore Window $\underline{\text { Help }}$ \\
\hline क्र & Histogram \\
\hline & Box Plot \\
\hline & Box Map \\
\hline & $\begin{array}{l}\text { Spatial Lag Bar Chart } \\
\text { Spatial Lag Pie Chart }\end{array}$ \\
\hline & $\begin{array}{l}\text { Moran Scatterplot Map } \\
\text { LISA Local Moran Map } \\
\text { G-Stat Map }\end{array}$ \\
\hline
\end{tabular}

Fig. 3.4. Contents of Explore Menu

text file and adding it as an additional Table (Import Table from Text File), or adding any SpaceStat Report file and joining it with an existing attribute table (Join SpaceStat Report File). The latter is a generic join function for any Report file generated by SpaceStat and is less efficient than the implementations specific to ESDA. Most of the functions in the Data menu are streamlined versions of standard ArcView operations (e.g., adding a Table) or of existing scripts from the Avenue script library (e.g., computing centroids). The streamlining is such that any unnecessary user input is avoided, in the sense that the SpaceStat conventions for file names and file structure are imposed or assumed. The only exception to this is the Weights from Shape File item, which invokes an external utility to carry out the transformation.

The Explore menu (Figure 3.4) is divided into three groups of commands. The first group contains tools to describe and visualize the spatial distribution of the data. Both the Histogram and Box Plot commands do not interact with SpaceStat but create ArcView "Charts" to represent the distribution of data (records in a field) in an active View. The histogram is standard and must be implemented in ArcView as a bar chart. It forms a non-spatial counterpart to a so-called "Equal Interval" classification in the View Legend Editor of ArcView. More precisely, the histogram shows the number of observations that fall in each category that corresponds to a given color (value interval) in an equal interval choropleth map. An additional tool button allows the identification of a selected histogram interval on the View. This is particularly useful when the View does not correspond to an equal interval classification, e.g., when it represents a quantile map. The histogram is only constructed for the selected spatial units in the View, so that it is possible to carry out a somewhat simplistic form of dynamic linking by constructing histograms for different spatial subsets of the data. However, each spatial subset in the View will require the explicit invocation of the histogram command and will result in a new chart (i.e., the selected data are not highlighted on the histogram for the complete data set, in contrast to what would happen in a standard implementation of brushing and linking). Also, while the select tool must be clicked on a given bar of the histogram to highlight the corresponding locations in the map, the bar itself is not highlighted, in contrast to common practice. 
In addition, earlier selections of spatial subsets are lost for future comparisons. These non-standard features are due to design constraints within ArcView. While they limit the extent of dynamic linking, they nevertheless provide a very useful way to visualize the spatial distribution of different subsets of the data in an interactive manner.

The Box Plot implementation is somewhat unusual in the sense that the four quartiles and the two sets of outliers (lower and upper) are visualized as a bar chart in ArcView. The height of the bars corresponding to each quartile is the same, except when outliers are present (the number of lower outliers and elements in the first quartile that are not outliers sum to the same total as the second and third quartile, and the same holds for the fourth quartile and upper outliers). The box plot can be constructed for any spatial subset of the data and the areal units corresponding to any bar can be highlighted in the View, in the same manner as for the histogram. In addition, a "graphic" is added to the View that provides a more traditional depiction of the box plot, including both median and mean of the data. This graphic can be moved around on the View in the same way as any other graphic object, but it cannot be used for interactive data analysis due to constraints in the ArcView design.

The Box Map command constructs a box map in the active View based on a joined Report File generated by SpaceStat. Since the file name for a box map Report File is always the same (boxmap.txt), no user intervention is needed to carry out the join. The only query involved is for the name of the variable that must be mapped.

The second group of commands in the Explore menu contains two functions to

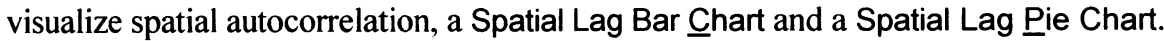
Both of these require a Report File from SpaceStat that contains the spatial lags for the variables of interest. As for the box map, this Report File has a fixed file name (sptran.txt) thereby avoiding the need for user interaction to carry out the join. However, the user is queried for the names of the variable and its associated spatial lag, as well as for the colors to represent them. These queries are shortened forms of the generic implementation of pie charts and bar charts as spot symbols on a View in the Avenue script library.

The last group of commands in the Explore menu deals more formally with local spatial association, in the form of a Moran Scatterplot Map (and associated Moran scatterplot as a chart), and maps highlighting the locations with significant values for the local Moran (LISA Local Moran Map) or G statistics (G-Stat Map). Each of these functions requires the input of a SpaceStat Report file with a fixed file name prefix (respectively MS , LM , or GI ) followed by the name of the spatial weights file for which the statistics were constructed. Again, user interaction is limited to queries for the file name and the variable of interest. The resulting maps are so-called "unique value" maps in the sense that each color corresponds with a unique value for the variable of interest (a quadrant in the Moran scatterplot or an indicator for the significance level of the LISA statistics). The interpretation of the resulting maps is straightforward.

In addition to the two extra menus, the ArcView interface is also augmented with two buttons: one to invoke SpaceStat (which can run as a true multitasked program in Windows 95 and Windows NT), the other to invoke the Dos command window. Three extra tool buttons are provided as well: one to select bars in the histogram and 
bar charts as outlined above, the two others to select spatial units within a given circle or within an arbitrary polygon. These tool buttons invoke slightly customized versions of scripts from the Avenue script library and are included to provide some degree of (albeit limited) dynamic linking between the different graphs.

\subsection{Illustration: The Spatial Pattern of Housing Values in West Virginia}

We illustrate the linked SpaceStat-ArcView environment with an initial exploration of the spatial pattern of housing values in West Virginia counties. The data are the median value of owner-occupied housing from the 1990 U.S. Census [Summary Tape File 1C, contained on the 1994 U.S. Counties CD Rom, U.S. Department of Commerce (1994)].

The spatial distribution of the housing values is illustrated in the four views represented in Figure 3.5. In the upper left corner is a familiar quintile map, to which the two histograms on the right are linked. The histogram on the bottom is for all West Virginia counties, the one on top only for those counties that border another

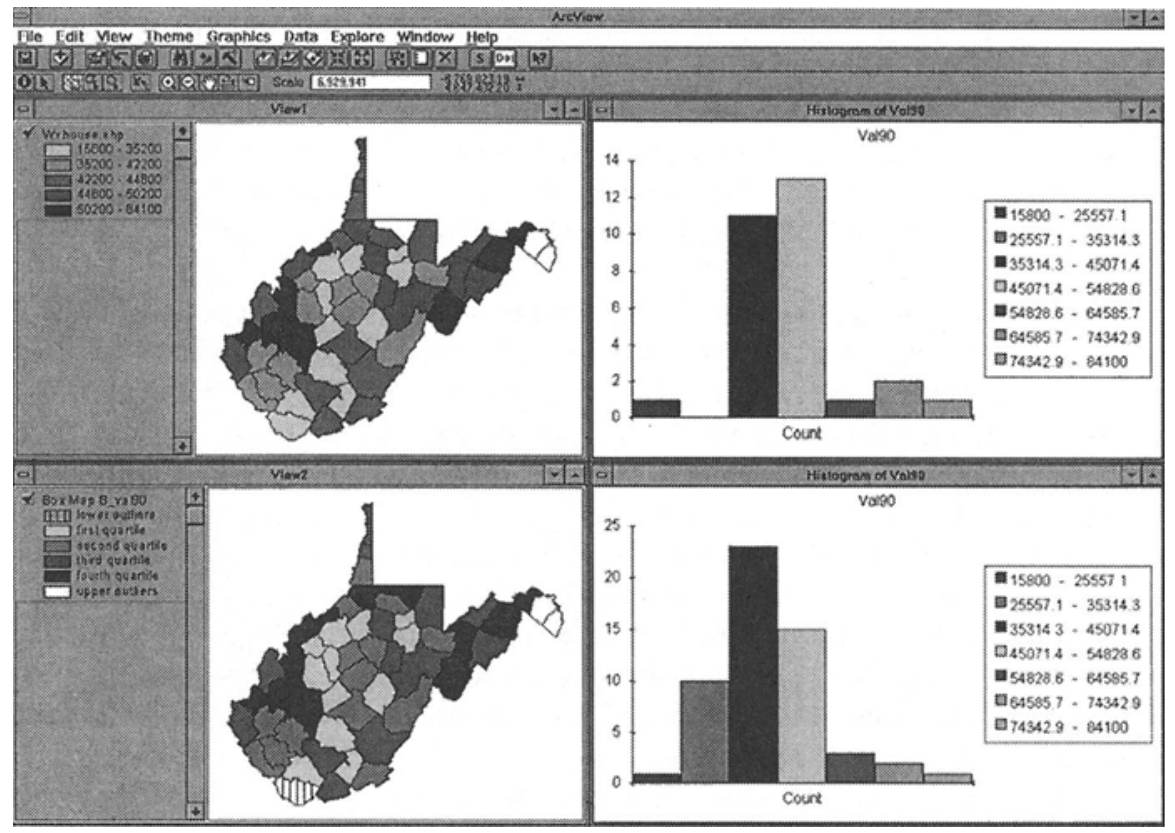

Fig. 3.5. Visualizing the Spatial Distribution of West Virginia Housing Values 
state (i.e., the counties on the outer rim of the state). The view in the bottom left is a box map for the housing values. The median value for the whole state is $\$ 44,000$, which falls in the interval that corresponds with the third bar in the histogram, illustrating the skewed nature of the distribution. In View 1, the three counties with the highest values are highlighted. This is obtained by clicking on the highest categories in the histogram on the bottom. Two of the counties are in the so-called Eastern Panhandle (Berkeley and Jefferson counties) while the third is Monongalia county, the location of West Virginia University. Interestingly, the highest value $(\$ 84,100)$ is for the easternmost county. The two panhandle counties are also singled out as upper outliers in the box map of View 2, but Monongalia county is not (its median of $\$ 64,600$ is well below 1.5 times the interquartile range of $\$ 11,300$ higher than the third quartile $-\$ 49,500)$. The box map also reveals a lower outlier in the southern part of the state (McDowell county, with a median value of $\$ 15,800$ ). An initial visual inspection of the two maps may suggest a systematic difference between the values in the inner core of the state and those at the outer rim. In order to assess the extent of this (in an exploratory fashion), a histogram is constructed for the "spatial selection" of the outer rim counties (upper chart). A comparison between the

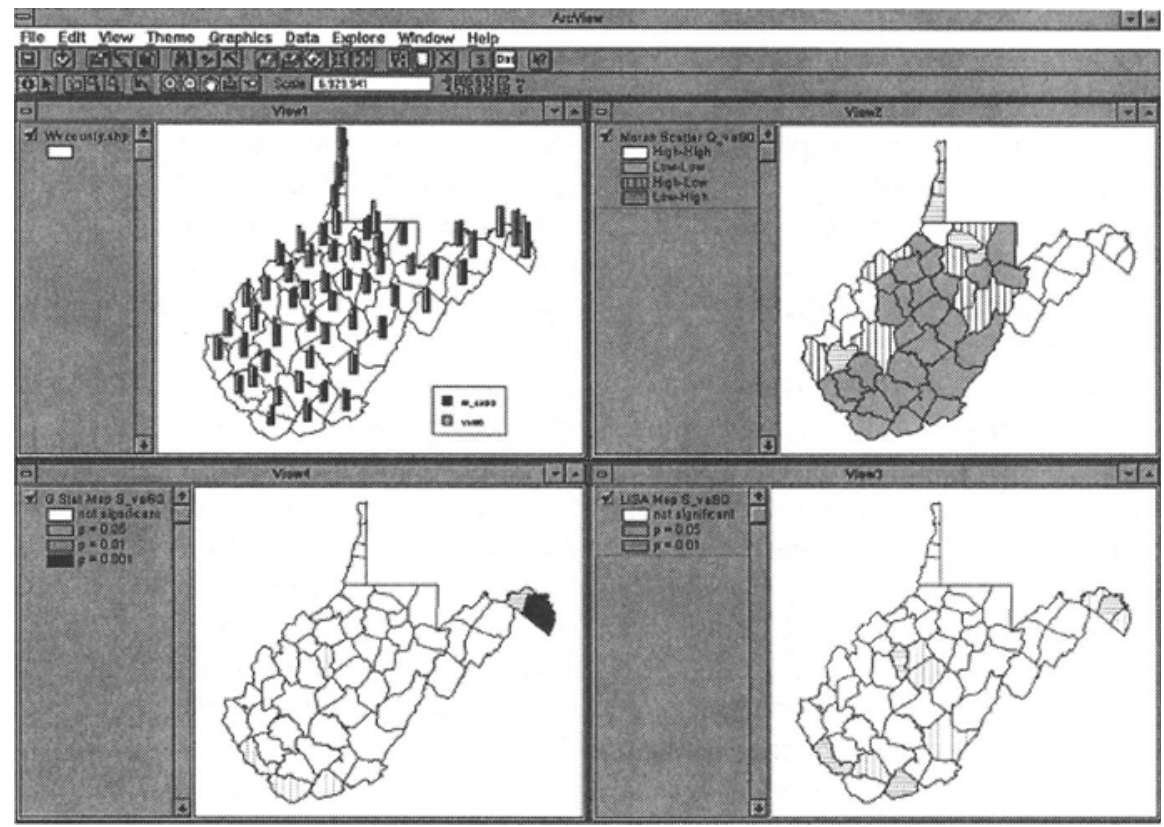

Fig. 3.6. Visualizing Spatial Association in West Virginia Housing Values 
two histograms indicates a stronger representation of higher values in the outer rim [note that the vertical scales in the two charts are not the same], with almost all the counties in the highest four histogram bars from the bottom chart included in the upper chart. This overall pattern is countered by the lone outlier at the low end which corresponds with the same county that also was identified in the box map (McDowell county is an outer rim county). Consequently, our initial hypothesis may need to be refined to exclude the southern rim counties from the high "spatial regime." In substantive terms, it turns out that the spatial pattern suggested by the views in Figure 3.5 could be associated with the influence of urbanization. While West Virginia itself is a highly rural state, a number of metropolitan areas are located close to its borders (except for its southern border), the most influential of which may be the Washington D.C. area near the eastern panhandle counties. This hypothesis would need to be assessed more rigorously, for example, by carrying out an adjusted spatial selection (excluding the southern counties from the outer rim regime) and constructing an indicator variable to be used for a spatial analysis of variance in SpaceStat.

Since the results of many statistical analyses (such as an analysis of variance) are affected by the presence of spatial autocorrelation, we assess global and local indicators of spatial association in Figure 3.6. The four views correspond with a spatial lag bar chart (View 1), a Moran scatterplot map (View 2), a map of locations with significant local Moran statistics (View 3) and a map of locations with significant $\mathrm{G}_{\mathrm{i}}{ }^{*}$ statistics (View 4). All measures were computed in SpaceStat, using a row-standardized first order contiguity weights matrix constructed from the shape file of the West Virginia counties. The global Moran's I statistic for the housing values is $\mathbf{0 . 4 6 0}$, corresponding to a highly significant standard normal z-value of 5.35 (using a randomization assumption). This global pattern is dominated by local indications of positive spatial association, as illustrated in Views 1 and 2. Overall, the spatial bar charts show very similar heights for each location and its spatial lag, with only a few notable spatial outliers. One of these is Monongalia county in the north, where the third largest median housing value (as indicated by the histogram in Figure 3.5) is surrounded by much lower values for the neighboring counties (i.e., a much shorter bar for the spatial lag in View 1). This is confirmed by the shading in the Moran scattermap (View 2), where Monongalia county is one of ten counties showing this pattern. However, the dominant pattern in the Moran scattermap is clearly associated with positive spatial autocorrelation between counties with low housing values, indicated by 27 counties in the low-low quadrant (13 counties are in the high-high quadrant). The smoothed picture represented by the scatterplot map suggests a cluster of low valued counties in the center and south of the state, with high valued counties in the northeast and along the western border. Only five counties are negative spatial outliers (low-high), located at the fringe of small clusters of higher valued counties. Clearly, not all these associations are "significant" and a query on the scattermap reveals that only three locations are outside the circle with radius equal to two standard deviations on the scatterplot (not illustrated here), the same three as indicated by the box map in Figure 3.5.

A more rigorous assessment of local spatial association is illustrated by the maps in View 3 and View 4 of Figure 3.6. While the local Moran and $\mathrm{G}_{\mathrm{i}}{ }^{*}$ statistics measure similar types of association, they are not identical, as indicated by the slight 
differences in significance between the two maps [see also Anselin (1995b) for further discussion and illustration of the differences and similarities]. All 10 counties with a significant local Moran statistic (based on a conditional permutation) are associated with positive spatial association, but only three of these (the three in the eastern panhandle of the state) indicate a cluster of high housing values, which can be seen from their location in the Moran scatterplot map in View 2. Similarly, two spatial clusters of low values can be distinguished, one in the center of the state (Calhoun, Gilmer and Braxton counties) and a string of three counties in the south (Mingo, Wyoming and Mercer counties). Three fewer counties are indicated with significant $\mathrm{G}_{i}{ }^{*}$ statistics, but except for one difference (McDowell county instead of Wyoming county in the south) all the others overlap with the ones in the LISA map. Again, the three eastern panhandle counties show a cluster of positive association (at a higher level of significance than in the LISA map) while the others show "negative" spatial association, in the sense of indicating clusters of low values.

In sum, the initial exploration of the spatial distribution and patterns of spatial association in West Virginia housing values suggests two main conclusions. One is the potential for the presence of two spatial regimes as mentioned above. The other is the persistent indication of several border counties as "outliers" in the sense that they possibly unduly influence the rest of the analysis. On the one hand, this may suggest that these counties do not fit the same pattern as the rest of the state. On the other hand however, this could be unduly influenced by a misspecification of the spatial weights for the border counties, which in the current example ignore neighbor counties outside the state (i.e., West Virginia is considered to be an "island"). Clearly, counties in the rim outside the state could be included in the analysis, which can easily be implemented in the linked SpaceStat-ArcView framework illustrated here.

\subsection{Future Directions}

The current implementation of the SpaceStat-ArcView linkage for exploratory spatial data analysis served two primary purposes: (a) to illustrate the types of ESDA techniques that can effectively be integrated within a GIS environment; and (b) to examine the potential and limitations of a loose coupling framework in a realistic setting. In terms of the first objective, we have demonstrated the importance of methods that deal explicitly with both global and local spatial autocorrelation in the data. In terms of the second, the interface between the two modules by means of file import and export is clearly limited and can be much improved by a tighter coupling. Two avenues for further development present themselves. One is to move SpaceStat to a full 32 bit Windows environment and allow spatial data analysis functions to be called from within ArcView (a similar setup could be implemented in a unix environment). Another, perhaps more effective approach would be to move away from a spatial analysis module as a single piece of software and to implement selected methods as spatial data analysis tools in small self-contained software applets, which can be invoked from ArcView using the Windows DLL conventions. Possibly, this is the most effective approach, since it would allow the individual user to customize 
the spatial data analysis "toolbox" for each application.

ArcView turned out to be a highly effective though limited environment to implement spatial data analysis. The Avenue language scripts, while extremely flexible, result in slow execution which severely limits the scope of analysis than can be carried out. For example, for data sets with hundreds of observations, the time required to compute and draw spatial bar charts or spatial lag pies becomes prohibitive. On the other hand, the linked SpaceStat-ArcView framework applied to small to medium sized examples (a hundred to a few hundreds of observations) is a powerful platform to teach the principles of ESDA within a GIS environment. We hope it will stimulate others to pursue the further integration of spatial data analysis techniques into such an environment.

\section{Acknowledgments}

The research reported on in this paper was supported in part by Grant SBR-9410612 from the U.S. National Science Foundation. The research was carried out while Shuming Bao was a Visiting Scholar at the Regional Research Institute, West Virginia University. Earlier versions of the paper were presented at the $30^{\text {th }}$ Anniversary Conference of the Regional Research Institute, Morgantown, WV, Nov. 6-8, 1995, and at the $92^{\text {nd }}$ Annual Meeting of the Association of American Geographers, Charlotte, NC, April 9-13. 1996.

The opinions expressed in the paper are solely those of the authors and do not imply an endorsement or any other support by MathSoft, Inc.

\section{References}

Anselin L. 1988. Spatial Econometrics: Methods and Models, Dordrecht: Kluwer Academic Anselin L. 1990. What is Special About Spatial Data? Alternative Perspectives on Spatial Data Analysis, in: D.A. Griffith, ed., Spatial Statistics, Past, Present and Future, Ann Arbor, MI:Institute of Mathematical Geography, pp. 63-77

Anselin L. 1992. SpaceStat: A Program for the Analysis of Spatial Data, National Center for Geographic Information and Analysis. University of California. Santa Barbara, CA

Anselin L. 1994. Exploratory Spatial Data Analysis and Geographic Information Systems, in:

M. Painho ed., New Tools for Spatial Analysis, Luxembourg: Eurostat, pp. 45-54

Anselin L. 1995a. SpaceStat Version 1.80 User's Guide, Regional Research Institute. West Virginia University, Morgantown, WV

Anselin L. 1995b. Local Indicators of Spatial Association - LISA, Geographical Analysis 27:93-115

Anselin L. 1996. The Moran Scatterplot as an ESDA Tool To Assess Local Instability in

Spatial Association. in: M. Fischer. H. Scholten and D. Unwin. eds.. Spatial Analytical Perspectives on GIS in Environmental and Socio-Economic Sciences. London: Taylor \& Francis

Anselin L. 1997a. Interactive Techniques and Exploratory Spatial Data Analysis, in: P. Longley. M. Goodchild. D. Maguire and D. Rhind (eds.), Geographical Information Systems: Principles, Techniques, Management and Applications, Cambridge: Geoinformation International

Anselin L.1997b. GIS Research Infrastructure for Spatial Analysis of Real Estate Markets. Journal of Housing Research 8

Anselin L. and Bao S. 1996. SpaceStat.apr User's Guide, Research Paper 9628, Regional Research Institute, West Virginia University, Morgantown. WV 
Anselin L. and Getis A. 1992. Spatial Statistical Analysis and Geographic Information Systems, The Annals of Regional Science 26:19-33

Anselin L., Dodson R. and Hudak S. 1993. Linking GIS and Spatial Data Analysis in Practice. Geographical Systems 1:3-23

Bailey T. C. 1994. A Review of Statistical Spatial Analysis in Geographical Information Systems, in: S. Fotheringham and P. Rogerson (eds.). Spatial Analysis and GIS. London: Taylor \& Francis, pp. 13-44

Bailey T. C. and Gatrell A.C. 1995. Interactive Spatial Data Analysis. Harlow: Longman Scientific and Technical

Bao S. and Henry M. 1996. Heterogeneity Issues in Local Measurements of Spatial Association, Geographical Systems 3:1-13

Bao S.. Henry M., Barkley D. and Brooks K. 1995. RAS: A Regional Analysis System Integrated with ARC/INFO, Computers, Environment and Urban Systems 18: 37-56

Barkley D., Henry M., Bao S. and Brooks K. 1995. How Functional are Economic Areas? Tests for Intra-Regional Spatial Association using Spatial Data Analysis, Papers in Regional Science 74:297-316

Batty M. and XieY. 1994. Modelling Inside GIS: Part I. Model Structures, Exploratory Spatial Data Analysis and Aggregation, International Journal of Geographical Information Systems 8:291-307

Becker R. and Cleveland W.S. 1987. Brushing scatterplots, Technometrics 29:127-142

Bradley R. and Haslett J. 1992. High Interaction Diagnostics for Geostatistical Models of Spatially Referenced Data, The Statistician 41:371-380

Buja A., Cook D. and Swayne D.F. 1996. Interactive High-Dimensional Data Visualization, Journal of Computational and Graphical Statistics 5:78-99

Buja A., McDonald J.A. , Michalak J. and Stuetzle W. 1991. Interactive Data Visualization using Focusing and Linking, in: G.M. Nielson and L. Rosenblum, eds., Proceedings of l'isualization '91, Los Alamitos, CA: IEEE Computer Society Press, pp. 155-162

Can A. 1996., Weight Matrices and Spatial Autocorrelation Statistics Using a Topological Vector Data Model, International Journal of Geographical Information Systems 10: 1009-1017

Cleveland W. S. 1993. Visualizing Data. Summit, NJ: Hobart Press

Cleveland W.S. and McGill M.E. 1988. Dynamic Graphics for Statistics, Pacific Grove, CA: Wadsworth

Cliff A.D. and Ord J.K. 1981. Spatial Processes: Models and Applications. London: Pion

Cook D.. Majure J.. Symanzik J. and Cressie N. 1996. Dynamic Graphics in a GIS: Exploring and Analyzing Multivariate Spatial Data Using Linked Software, Computational Statistics 11:467-480

Cressie N. 1993. Statistics for Spatial Data, New York: Wiley

Ding Y. and A. S. Fotheringham The Integration of Spatial Analysis and GIS. Computers, Environment and Urban Systems 16 3-19 1992.

ESRI Avenue, Customization and Application Development for Arcliew, Redlands, CA: Environmental Systems Research Institute. 1994.

ESRI Arcl'iew 2.1 The Geographic Information System for Everyone Redlands CA: Environmental Systems Research Institute 1995a.

ESRI Understanding GIS, The ARC/INFO Method, Redlands, CA: Environmental Systems Research Institute 1995b.

ESRI Arcliew l'ersion 2 Shapefile Technical Description. White Paper. Redlands. CA: Environmental Systems Research Institute. 1995c.

Farley J.A., W.F. Limp, and J. Lockhart. The Archeologist's Workbench: Integrating GIS. Remote Sensing. EDA and Database Management. in: K. Allen. F. Green and E. Zubrow 
(eds.), Interpreting Space: GIS and Archaeology, London: Taylor \& Francis, pp. 141-164, 1990.

Fischer M. M. and Nijkamp P. 1993. Geographic Information Systems, Spatial Modelling and Policy Evaluation, Berlin: Springer-Verlag

Fischer M.M., Scholten H. and Unwin D. 1996. Spatial Analytical Perspectives on GIS in Environmental and Socio-Economic Sciences, London: Taylor \& Francis, 1996

Flowerdew R. and Green M. 1991. Data Integration: Statistical Methods for Transferring Data Between Zonal Systems, in: I. Masser and M. Blakemore (eds.), Handling Geographical Information, London: Longman, pp. 38-54

Fotheringham A. S. and Charlton M. 1994. GIS and Exploratory Spatial Data Analysis: An Overview of some Research Issues, Geographical Systems 1:315-327

Fotheringham A. S. and Rogerson P. 1994. Spatial Analysis and GIS, London: Taylor \& Francis

Getis A. and Ord K. 1992. The Analysis of Spatial Association by Use of Distance Statistics, Geographical Analysis 24:189-206

Good I.J. 1983. The Philosophy of Exploratory Data Analysis, Philosophy of Science 50:283-295

Goodchild M. F. 1987. A Spatial Analytical Perspective on Geographical Information Systems, International Journal of Geographical Information Systems 1:327-334

Goodchild M. F. 1992. Geographical Information Science, International Journal of Geographical Information Systems 6:31-45

Goodchild M. F., Haining R.P., Wise S., et al. 1992. Integrating GIS and Spatial Analysis Problems and Possibilities, International Journal of Geographical Information Systems 6:407-423

Griffith D.A. 1993. Which Spatial Statistics Techniques Should Be Converted to GIS Functions? in: M.M. Fischer and P. Nijkamp (eds.), Geographic Information Systems, Spatial Modelling and Policy Evaluation. Berlin: Springer-Verlag, pp. 101-114

Haining R. 1990. Spatial Data Analysis in the Social and Environmental Sciences, Cambridge: Cambridge University Press

Haining R. 1994. Designing Spatial Data Analysis Modules for Geographical Information Systems, in: S. Fotheringham and P. Rogerson (eds.). Spatial Analysis and GIS, London: Taylor \& Francis, pp. 45-63

Haslett J. 1992. Spatial Data Analyis - Challenges, The Statistician 41:271-284

Haslett J. and Power G.M. 1995. Interactive Computer Graphics for a more Open Exploration of Stream Sediment Geochemical Data, Computers and Geosciences 21:77-87

Haslett J., Wills G. and Unwin A. 1990. SPIDER - An Interactive Statistical Tool for the Analysis of Spatially Distributed Data, International Journal of Geographical Information Systems 4:285-296

Haslett J.. Bradley R., Craig P., Unwin A. and Wills C. 1991. Dynamic Graphics for Exploring Spatial Data with Applications to Locating Global and Local Anomalies. The American Statistician 45:234-242

Lam N., Fan M. and Liu K-B. 1996. Spatial-Temporal Spread of the AIDS Epidemic, 1982-1990: A Correlogram Analysis of Four Regions of the United States, Geographical Analysis 28:93-107

MacDougall E.B. 1991. A Prototype Interface for Exploratory Analysis of Geographic Data. Proceedings of the Eleventh Annual ESRI User Conference, vol. 2.. Redlands, CA: Environmental Systems Research Institute. Inc., pp. 547-553

Majure J. and Cressie N. 1997. Dynamic Graphics for Exploring Spatial Dependence in Multivariate Spatial Data, Geographical Systems (forthcoming)

Majure J., Cook D.. Cressie N.. Kaiser M.. Lahiri S. and Symanzik J. 1996. Spatial CDF 
Estimation and Visualization with Applications to Forest Health Monitoring, Computing Science and Statistics 27:93-101

Majure J.. Cressie N., Cook D. and Symanzik J. 1996. GIS, Spatial Statistical Graphics, and Forest Health, in: Proceedings, Third International Conference/Workshop on Integrating GIS and Environmental Modeling, Santa Fe, NM, January 21-26, 1996, Santa Barbara, CA, National Center for Geographic Information and Analysis (CD ROM)

MathSoft. 1996a. S+Gislink, Seattle: MathSoft, Inc.

MathSoft. 1996b. S+Spatialstats User's Manual, Version 1.0, Seattle: MathSoft, Inc.

Monmonier M. 1989. Geographic Brushing: Enhancing Exploratory Analysis of the Scatterplot Matrix, Geographical Analysis 21:81-84

Oden N.L. 1984., Assessing the Significance of a Spatial Correlogram, Geographical Analysis 16:1-16

O'Loughlin J. and Anselin L. 1996. Geo-Economic Competition and Bloc Formation: U.S., German and Japanese Trade Development, 1968-1992, Economic Geography 72:131-160 Openshaw S. 1991. Developing Appropriate Spatial Analysis Methods for GIS, in: D. Maguire, M.F. Goodchild and D. Rhind (eds.), Geographical Information Systems: Principles and Applications, Vol 1, London: Longman, pp. 389-402

Openshaw S. and Fischer M.M. 1995. A Framework for Research on Spatial Analysis Relevant to Geo-Statistical Information Systems in Europe, Geographical Systems 2:325-337

Ord J. K. and Getis A. 1995. Local Spatial Autocorrelation Statistics: Distributional Issues and Applications, Geographical Analysis 27:286-306

Painho M. 1994. New Tools for Spatial Analysis, Luxembourg: Eurostat

Stuetzle W. 1987. Plot windows, Journal of the American Statistical Association 82:466-475

Symanzik J., Majure J., Cook D. and Cressie N. 1994. Dynamic Graphics in a GIS: A Link between Arc/Info and XGobi, Computing Science and Statistics 26:431-435

Symanzik J., Majure J. and Cook D. 1996. Dynamic Graphics in a GIS; A Bidirectional Link between ArcView 2.0 and XGobi, Computing Science and Statistics 27:299-303

Talen E. 1997. Visualizing Fairness: Equity Maps for Planners, Journal of the American Planning Association (forthcoming)

Talen E. and Anselin L. 1997. Assessing Spatial Equity: The Role of Access Measures. Environment and Planning $A$ (forthcoming)

Tukey J.W. 1977. Exploratory Data Analysis, Reading MA: Addison-Wesley

Unwin A. 1994. REGARDing Geographic Data, in: P. Dirschedl and R. Osterman (eds.). Computational Statistics, Heidelberg: Physica Verlag, pp. 345-354

Unwin A. 1996. Exploratory Spatial Analysis and Local Statistics, Computational Statistics 11:387-400

Upton G. J. and Fingleton B. 1985. Spatial Data Analysis by Example, New York: Wiley

U.S. Department of Commerce.1994. USA Counties 1994 CD-ROM, Washington, D.C.: Bureau of the Census

Venables W. N and Ripley B.D. 1994. Modern Applied Statistics with S-Plus, New York: Springer-Verlag

Williams I.. Limp W.. Briuer F. 1990. Using Geographic Information Systems and Exploratory Data Analysis for Archeological Site Classification and Analysis, in: K. Allen, F. Green and E. Zubrow (eds.). Interpreting Space: GIS and Archaeologv, London: Taylor \& Francis, pp. 239-273

Zhang A., Yu H. and Huang S. 1994. Bringing Spatial Analysis Techniques Closer to GIS Users: A User-Friendly Integrated Environment for Statistical Analysis of Spatial Data, in: T. C. Waugh and R. G. Healy (eds.), Advances in GIS Research. London: Taylor \& Francis, pp. 297-313 